\title{
Wavelength Division Multiplexing Scheme for Radio-Frequency Single Electron Transistors
}

\author{
T. R. Stevenson, F. A. Pellerano, and C. M. Stahle \\ NASA Goddard Space Flight Center, Codes 553 and 555, Greenbelt, MD 20771, USA
}

K. Aidala and R. J. Schoelkopf

Department of Applied Physics, Yale University, PO Box 208284, New Haven, CT 06520-8284, USA

\begin{abstract}
We describe work on a wavelength division multiplexing scheme for radio-frequency single electron transistors. We use a network of resonant impedance matching circuits to direct applied $\mathrm{rf}$ carrier waves to different transistors depending on carrier frequency. Using discrete components, we made a two-channel demonstration of this concept and successfully reconstructed input signals with small levels of cross coupling. A lithographic version of the $\mathrm{rf}$ circuits had measured parameters in agreement with electromagnetic modeling, with reduced cross capacitance and inductance, and should allow 20 to 50 channels to be multiplexed.
\end{abstract}

\section{INTRODUCTION}

Several types of superconducting photodetectors are being developed for astronomical applications. ${ }^{1,2,3}$ Despite cryogenic operation, such detectors are desirable because of capabilities such as single-photon spectroscopy, or extreme levels of sensitivity. Large format arrays will require integration with multiplexed on-chip amplifiers. Multiplexing schemes ${ }^{4}$ have been developed for low impedance detectors ${ }^{1}$ with dc SQUID amplifiers. For high impedance detectors, ${ }^{2,3}$ the Radio-Frequency Single Electron Transistor (RF-SET) ${ }^{5}$ seems well suited as a amplifier. ${ }^{6}$ Here we describe experimental demonstrations and theoretical limits of a Wavelength Division Multiplexing (WDM) scheme ${ }^{6}$ in which a network of resonant circuits sorts and directs $\mathrm{rf}$ power, simultaneously applied at many carrier frequencies, to individually power and probe a collection of RF-SETs using a single rf following amplifier.

\section{RF READOUT AND MULTIPLEXING SCHEME}

Single Electron Transistors (SETs) are cryogenic quantum-effect devices which utilize quantization of charge on a small conducting island to yield a very high performance electrometer. ${ }^{7}$ An SET amplifier dissipates only picowatts of power and has sub-femtofarad input capacitance. An RF-SET obtains greatly increased readout 
bandwidth, over $100 \mathrm{MHz}$, by using an rf readout technique and a resonant LC circuit to better match the SET's $\approx 100 \mathrm{k} \Omega$ output impedance to a following amplifier.

To multiplex RF-SETs, we use a scheme similar to one used for RF-SQUIDs. ${ }^{8}$ We connect the following amplifier via a coaxial line to a parallel combination of $\mathrm{LC}$ circuits, one for each SET [see Fig. 1(a)]. As for a single RF-SET, a directional coupler is used to apply rf carrier power to the LC circuits while monitoring reflected power with a cryogenic HEMT amplifier. Each LC circuit has a unique resonance frequency, and instead of a single frequency carrier wave, a frequency comb is generated with a component for each resonance. Gate signals modify SET output impedances, and changes in reflected powers can be detected and demultiplexed with room temperature rf electronics. Simultaneous readout of all channels is possible.
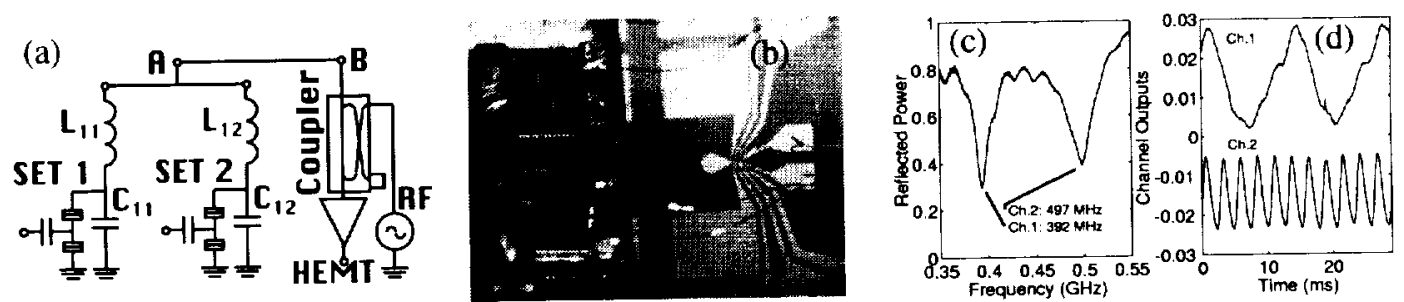

FIGURE 1. (a) Circuit for wavelength division multiplexing of RF-SETs. (b) Two-channel demonstration using wire coils bonded to pads on SET chip. (c) Frequency dependence of reflected power ratio with SETs in high/low impedance states. (d) Demultiplexed SET signals.

Efficient coupling of carrier power to the $i^{\text {th }}$ SET, and sensitive detection of its reflected signal, depend on transforming its output impedance $R_{i}$ down close to the $R_{c}$ $=50 \Omega$ input and noise impedance of the HEMT. The L-section circuit ${ }^{9}$ consisting of $C_{1 i}$ in parallel, and $L_{1 \mathrm{i}}$ in series, with $R_{i}$ transforms $R_{i}$ to a small effective resistance value $r_{i}=R_{i} / Q_{T}^{2}$ at point $A$ in Fig. 1(a), where $Q_{T}^{-1}=\left(L_{1 i} / C_{1 i}\right)^{1 / 2} / R_{i}$ is the fractional bandwidth over which the transformation is effective around the resonance frequency $\omega_{1 i}=\left[\left(1-Q_{T}^{-2}\right) /\left(L_{1 i} C_{1 i}\right)\right]^{1 / 2}$. If the SETs are biased to a common value $R_{i}=R_{s}$, then a common bandwidth $Q_{T}^{-1}=\left(R_{c} / R_{s}\right)^{1 / 2}$ and ratio $L_{1 i} / C_{1 i}=R_{c} R_{s}$ is required for all channels. The parallel circuits have modest interaction since, at a given carrier frequency, one circuit is nearly a short, while others have large reactances.

\section{TWO-CHANNEL DEMONSTRATION}

To investigate this rf multiplexing scheme using two channels, we wirebonded two $\approx 200 \mathrm{nH}$ chip inductors ${ }^{10}$ to a pair of SETs on a silicon chip [see Fig. 1(b)]. The other ends of the inductors were bonded to an SMA microstrip launcher and coax leading from the $250 \mathrm{mK}$ sample stage on a ${ }^{3} \mathrm{He}$ refrigerator to a directional coupler and HEMT amplifier ${ }^{11}$ at $4 \mathrm{~K}$. The $C_{1 i} \approx 0.5 \mathrm{pF}$ consisted of pad capacitances to ground.

The rf power reflected from the parallel combination of the two tank circuits is shown versus frequency in Fig. 1(c). The two resonance frequencies were 392 and $497 \mathrm{MHz}$. Figure 1(d) shows in the time domain the demultiplexed outputs of the two RF-SETs responding to different gate signals. The open-loop response to the ramping gate signals was approximately sinusoidal for each RF-SET. With Fourier analysis of 
the Fig. 1(d) data, we found an $8 \%$ coupling of $\mathrm{Ch} .2$ in the $\mathrm{Ch} .1$ output, and $4 \%$ of Ch. 1 in Ch.2. This effect, where impedance changes of one SET off-resonance perturb reflection of the carrier allocated for a different SET, will be diminished when feedback is used to fix each SET's operating point. Closed-loop coupling will depend on gate cross-capacitance, which we can reduce by adding appropriate guard traces.

(a)

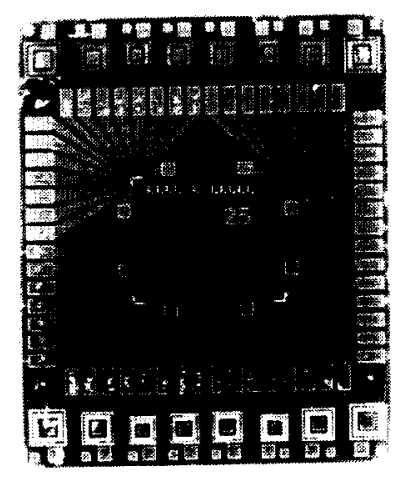

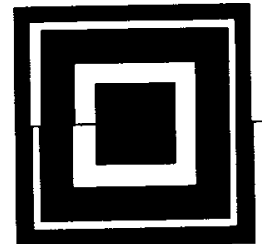

(b)
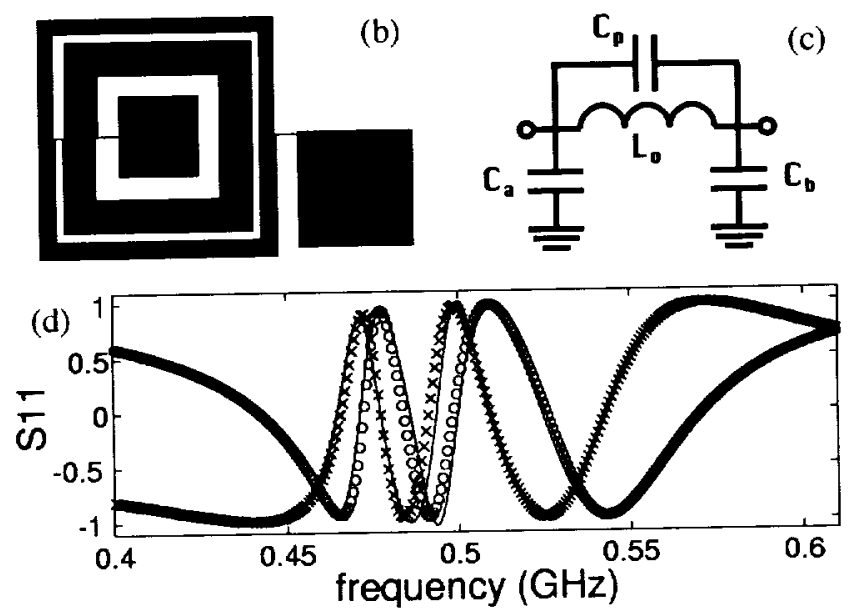

FIGURE 2. (a) Lithographic rf circuits: 16 inductors of 5 designs are at top and bottom; 68 bonding pads connecting to e-beam fields serve as capacitors $C_{I_{i}}$. (b) Geometry to minimize cross coupling (smallest coil, $619 \mu \mathrm{m}$ outer side). (c) Parametric inductor model. (d) Real (o) and imaginary (x) parts of measured s-parameter for a three-circuit array, and fitted model curves (solid lines).

\section{LITHOGRAPHIC TANK CIRCUITS}

Producing arrays of tank circuits by hand selection and assembly of components clearly has limitations. We next patterned circuits by optical lithography directly on substrates for SET fabrication by electron-beam lithography [see Fig. 2(a)]. We used planar 3D electromagnetic modeling software ${ }^{12}$ to design circuits with desired components and minimimal cross inductance and capacitance. Figure 2(b) shows the coil geometry, with $N_{o}$ outer turns and $2 N_{o}$ counter-wound inner turns of equal total areas, which we chose to reduce mutual inductance between coils to $<1 \%$. Since coil internal resonances were $>1 \mathrm{GHz}$ by design, we found we could accurately describe our numerical modeling of a tank circuit by the lumped element model in Fig. 2(c).

To test our rf modeling, we made calibrated measurements of the reflection coefficient of unloaded circuit arrays with coils superconducting at $4 \mathrm{~K}$. Figure 2(d) shows results for three circuits in parallel. Measured $\omega_{1 i}$ were about $5 \%$ higher than designed. The circuits were lossless to measurement precision with $Q>500$. Fitting data, we found capacitances and inductances within $\approx 10 \%$ of our predictions, and estimated cross-capacitance was $\approx 2 \%$ or less, consistent with our calculations.

The lithographic circuits have $\left(L_{1 i} / C_{1 i}\right)^{1 / 2} \approx 670 \Omega$, and over-transform a typical $R_{s}$ $\approx 50 \mathrm{k} \Omega$ to $9 \Omega$ instead of $50 \Omega$, with a factor of two degradation in coupling to the SET, from $100 \%$ to $50 \%$. We did this to increase the number of channels we can multiplex within the octave bandwidth $(300-600 \mathrm{MHz})$ of our HEMT amplifier. We 
find sufficiently small open-loop coupling (nulled in closed-loop) exists between rf channels separated by $\Delta \omega>2 \omega_{1 i} / Q_{T}$. That limits the number of channels to $N \approx Q_{T} / 3$ in an octave bandwidth, giving $N \approx 25$ instead of 11 when transforming to $9 \Omega$ instead of $50 \Omega$. With another circuit added to compensate for over-transformed impedances, we calculate that $N=50$ is possible without sacrificing power coupling. ${ }^{13}$

\section{CONCLUSION}

We have demonstrated the ability to both supply power to and multiplex outputs of many SETs using only a single rf connection. Our technique exploits a network of resonant impedance matching circuits which directs applied carrier waves to different SETs depending on frequency. Using optical lithography and electromagnetic modeling, we fabricated circuits capable of multiplexing 20 to 50 channels. Such a system would provide a compact readout for superconducting photodetector arrays.

\section{ACKNOWLEDGMENTS}

The authors thank Peter Wahlgren, Abdelhanin Aassime, and Per Delsing of Chalmers University for SET fabrication, and Richard Bradley for the HEMT.

\section{REFERENCES}

1. R. W. Romani, R. W. Miller, B. Cabrera, E. Figueroa-Feliciano, and S. W. Nam, Astrophys. J. 521, L1 53 (1999); K. D. Irwin, G. C. Hilton, J. M. Martinis, S. Deiker, N. Bergren, S. W. Nam, D. A. Rudman, and D. A. Wollman, Nucl. Instrum. Meth. A 444, 184 (2000); N. Tralshawala, R. P. Brekosky, M. J. Li, E. Figueroa-Feliciano, F. M. Finkbeiner, M. A. Lindeman, C. M. Stahle, and C. K. Stahle, IEEE Trans. Appl. Supercond. 11, 755 (2001).

2. A. Peacock, P. Verhoeve, N. Rando, A. van Dordrecht, B. G. Taylor, C. Erd, M. A. C. Perryman, R. Venn, J. Howlett, D. J. Goldie, J. Lumley, and M. Wallis, Nature 381, 135 (1996).

3. R. J. Schoelkopf, S. H. Moseley, C. M. Stahle, P. Wahlgren, P. Delsing, IEEE Trans. Appl. Supercond. 9, 2935 (1999).

4. J. A. Chervenak, E. N. Grossman, K. D. Irwin, J. M. Martinis, C. D. Reintsema, C. A. Allen, D. I. Bergman, S. H. Moseley, and R. Shafer, Nucl. Instrum. Meth. A 444, 107 (2000); J. Yoon, J. Clarke, J. M. Gildemeister, A. T. Lee, M. J. Myers, P. L. Richards, and J. T. Skidmore, Appl. Phys. Lett. 78, 371 (2001).

5. R. J. Schoelkopf, P. Wahlgren, A. A. Kozhevnikov, P. Delsing, D. E. Prober, Science 280, 1238 (1998).

6. T. R. Stevenson, A. Aassime, P. Delsing, R. Schoelkopf, K. Segall, C. M. Stahle, IEEE Trans. Appl. Supercond. 11, 692 (2001).

7. T. A. Fulton, G. J. Dolan, Phys. Rev. Lett. 59, 109 (1987).

8. M. Muck, IEEE Trans. Mag. 27, 2986 (1991).

9. L. Weinberg, Network Analysis and Synthesis, New York, McGraw-Hill, 1962.

10. Part no. 0805CS-221XKBC, Coilcraft, 1102 Silver Lake Road, Cary, IL 60013, USA.

11. Our HEMT amplifier was provided by the National Radio Astronomy Observatory, 2015 Ivy Road, Suite 219, Charlottesville, VA 22903, USA.

12. SONNET $B$, Sonnet Software, Inc. 1020 Seventh North St., Suite 210, Liverpool, NY 13088, USA.

13. T. R. Stevenson, F. A. Pellerano, C. M. Stahle, K. Aidala, and R. J. Schoelkopf, Appl. Phys. Lett. (submitted). 